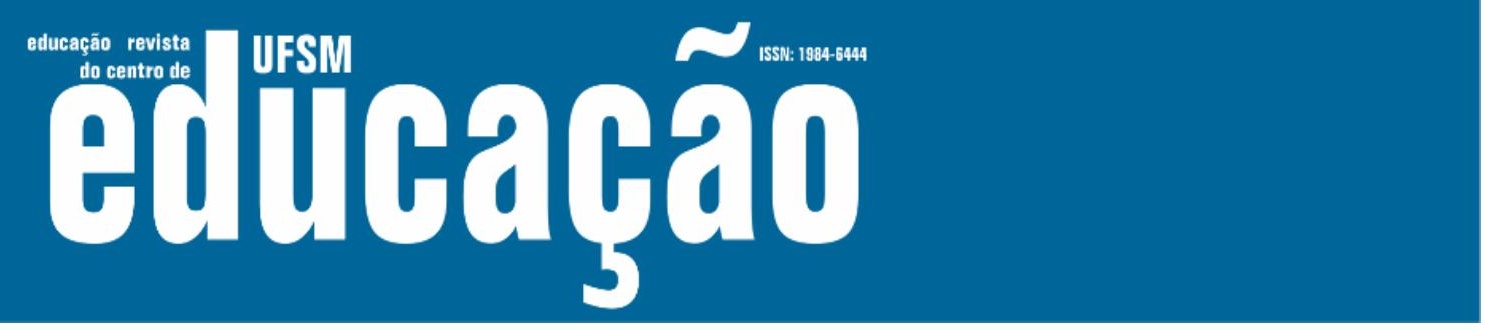

ISSN: 1984-6444 | http://dx.doi.org/10.5902/1984644443450

\title{
Espiritualidade no ensino: a perspectiva dos discentes de uma universidade pública da área da saúde
}

\author{
Teaching Spirituality: the perspective of the students of a health public \\ university
}

Lahanna da Silva Ribeiro

Enfermeira pela Universidade Federal de Ciências da Saúde de Porto Alegre. Porto Alegre, Rio Grande do Sul, Brasil.

lahannas@gmail.com - orcid.org/0000-0003-0219-7348

Émilly Giacomelli Bragé

Enfermeira pela Universidade Federal de Ciências da Saúde de Porto Alegre. Porto Alegre, Rio Grande do Sul, Brasil.

emilly.ufcspa@gmail.com - orcid.org/0000-0001-6970-8320

Bruna Luísa Ribeiro de Almeida

Graduanda da Universidade Federal de Ciências da Saúde de Porto Alegre. Porto Alegre, Rio Grande do Sul, Brasil.

bruninaribeiro@hotmail.com - orcid.org/0000-0002-5298-3665

Eliane Goldberg Rabin

Professora Adjunta da Universidade Federal de Ciências da Saúde de Porto Alegre. Porto Alegre, Rio Grande do Sul, Brasil.

elianerabin@gmail.com - orcid.org/0000-0003-1450-2012

Recebido em 21 de maio de 2020

Aprovado em 07 de janeiro de 2021

Publicado em 30 de dezembro de 2021

\section{RESUMO}

A espiritualidade é uma parte complexa e multidimensional da experiência humana, com base inerente de cada pessoa do significado e do propósito de vida, encontrada em todas as culturas. A consideração do ser humano de forma integral, representa um aspecto importante da formação dos profissionais da saúde. Tem crescido o reconhecimento da importância da espiritualidade na saúde, mas pouco se ensina ou se aplica na prática aos profissionais desta área. Os cursos de graduação e de pósgraduação, na sua grande maioria, não desenvolvem conteúdos relacionados à espiritualidade nos seus currículos. Assim, o objetivo do estudo foi investigar a compreensão dos discentes da Universidade Federal de Ciências da Saúde de Porto Alegre a respeito do tema espiritualidade na educação e na saúde, por meio da análise de presença ou ausência da abordagem do tema espiritualidade no currículo acadêmico, e de como estes avaliam a pertinência do tema. Trata-se de um estudo 


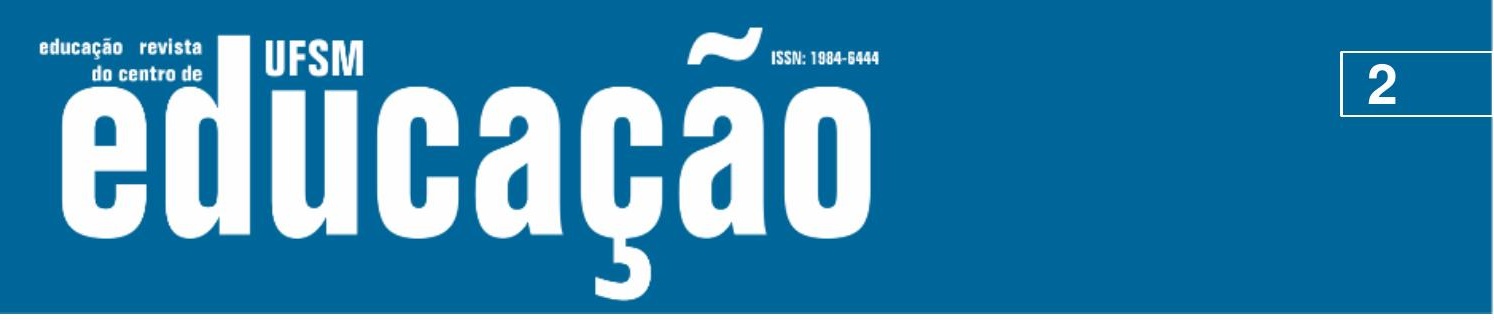

ISSN: 1984-6444 | http://dx.doi.org/10.5902/1984644443450

transversal que tem como população os discentes dos Cursos de Graduação e PósGraduação da Universidade Federal de Ciências da Saúde de Porto Alegre. Realizouse a coleta de outubro de 2016 a novembro de 2019, utilizando-se questionários em formato impresso. Um total de 632 alunos responderam ao questionário, e 65,7\% destes entendem que é pertinente abordar aspectos relacionados à "espiritualidade" na formação acadêmica. Conclui-se que os discentes dos diferentes cursos e séries reconhecem a relevância do estudo da espiritualidade na saúde, entretanto, a carência deste conteúdo durante a formação acadêmica resulta em desconhecimento e insegurança para abordar o tema com os pacientes.

Palavras-chave: Espiritualidade; Ensino; Educação.

\section{ABSTRACT}

Spirituality is a complex and multidimensional part of the human experience, with each person's inherent basis of the meaning and purpose of life, found in all cultures. The consideration of the human being in an integral way, represents an important aspect of health professionals training. The recognition of the importance of spirituality in health has grown, but little is taught or applied for professional practice. The vast majority of undergraduate and graduate courses do not develop content related to spirituality in their curricula. The aim of this study was to investigate the understanding of students at the Federal University of Health Sciences of Porto Alegre, regarding to the theme spirituality in education and health, through the analysis of the presence or absence of the theme in the academic curriculum and how they assess the relevance of the theme. This is a cross-sectional study with the population of undergraduate and graduate students at the Federal University of Health Sciences of Porto Alegre. The collection took place from October 2016 to November 2019, using questionnaires in printed format. A total of 632 students answered the questionnaire, and $65.7 \%$ of whom believe that it is pertinent to address aspects related to "spirituality" in academic training. It is concluded that students from different courses and grades recognize the relevance of the study of spirituality in health, however, the lack of this content during academic training results in ignorance and insecurity to approach the topic with patients.

Keywords: Spirituality; Teaching; Education. 


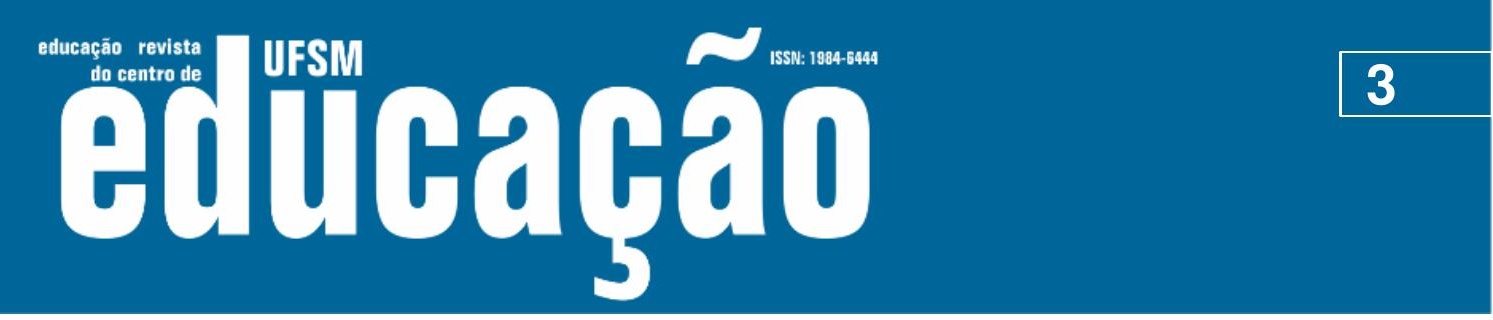

ISSN: 1984-6444 | http://dx.doi.org/10.5902/1984644443450

\section{Introdução}

Sabe-se que o conceito de espiritualidade varia conforme a nação e a crença, em decorrência da sua multiplicidade pelo mundo (OLIVEIRA, 2017). Tem-se na literatura que a espiritualidade é uma parte complexa e multidimensional da experiência humana, com base inerente de cada pessoa do significado e do propósito de vida, encontrada em todas as culturas (KOENIG, 2012). Em contraponto, a religiosidade consiste na forma com que uma pessoa crê, acompanha e coloca em ação a religião específica.

A religião é um sistema organizado de crenças, práticas, rituais e símbolos destinados a facilitar a proximidade com o sagrado e o transcendente compartilhadas por uma comunidade organizada (KOENIG, 2001). Refere-se ao Cristianismo, Judaísmo, Islamismo, Hinduísmo, Budismo e outras tradições religiosas com suas diversas vertentes.

Religiosidade diz respeito ao nível de envolvimento religioso e o reflexo desse envolvimento na vida da pessoa, ou seja, o quanto isso influencia seu cotidiano, seus hábitos e sua relação com o mundo (KOENIG, 2001). Pode ser organizacional: participação no templo religioso; ou não organizacional: rezar, ler livros, assistir programas religiosos na televisão (MOREIRA-ALMEIDA, NETO, KOENIG, 2006). O papel fundamental da religiosidade não é prover um código moral, mas sim mediar o encontro com o sagrado (SULMASY, 2009). A religiosidade é classificada em intrínseca e extrínseca: a primeira refere-se à quando há a centralidade na vida do indivíduo, e a segunda é quando se utiliza a religião para obter vantagem sobre pessoas, bens materiais ou instituições (GUSSI E DYTZ, 2008).

É importante considerar que existe relação entre religiosidade e espiritualidade, no entanto, estas não dependem uma da outra. Logo, para o exercício da espiritualidade é dispensável que haja prática religiosa, é preciso somente que a ação envolva o espiritual, a alma, o que vai além da religião (SOUZA, MAFTUM E BAIS, 2008).

Dessa forma, traz-se a contribuição da Association of American Medical 


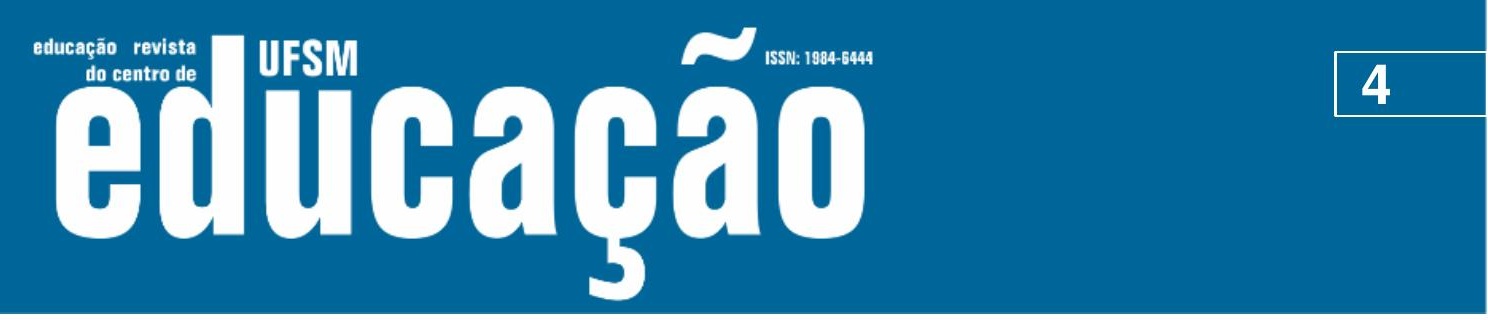

ISSN: 1984-6444 | http://dx.doi.org/10.5902/1984644443450

Colleges (AAMC) (1998) sobre o tema:

\begin{abstract}
A espiritualidade é reconhecida como um fator que contribui para a saúde de muitas pessoas. O conceito de espiritualidade é encontrado em todas as culturas e sociedades. Ela é expressa nas buscas individuais para um sentido último através da participação na religião e ou crença em Deus, família, naturalismo, racionalismo, humanismo, e nas artes. Todos esses fatores podem influenciar na maneira como os pacientes e os cuidadores profissionais da saúde percebem a saúde e a doença e como eles interagem uns com os outros (ASSOCIATION OF AMERICAN MEDICAL COLLEGES, 1998).
\end{abstract}

Diferentes estudos trazem a espiritualidade como a junção de práticas, realizações e percepções de si mesmos e do próximo causando uma interferência no processo saúde-doença, tanto do profissional de saúde quanto do paciente. (OLIVEIRA, 2017; KOENIG, 2012; SÁ, 2009). O bem-estar espiritual melhora a função imunológica, reduz significativamente 0 estresse relacionado a patologias degenerativas e neurológicas, além de ser um fator preponderante na qualidade de vida. Outros benefícios reconhecidos são o aumento do vigor em doentes crônicos, a melhora nos desfechos de reabilitação de vítimas com traumatismo cerebral, a redução do tempo de hospitalização e o aumento da resiliência de pacientes e cuidadores (DAL-FARRA, 2010; SAAD, 2001).

A necessidade de inserir conteúdos relacionados à espiritualidade nos currículos dos cursos da área da saúde, está relacionada a sua comprovada influência nos resultados do tratamento e na manutenção da saúde dos pacientes, cuidadores e profissionais. No entanto, somente algumas instituições dos Estados Unidos e do Reino Unido, que são responsáveis pela maior produção de estudos na área, conseguiram integrar o tema à graduação. No Brasil, as ações em prol da inclusão deste tema nos currículos são iniciais, e nos poucos exemplos existentes se observa a realização de cursos que utilizam este conteúdo para a oferta de disciplinas optativas. (ESPINHA et al, 2013; LUCCHETTI et al, 2011; LUCCHETTI, 2013).

No ano de 2002, a Organização Mundial de Saúde (OMS) definiu saúde como além da ausência da enfermidade, ou seja, é uma combinação de bem-estar físico, psíquico, social e espiritual. Dessa forma, evidencia-se que a espiritualidade deve ser reconhecida como parte do cuidado e abordada na formação dos profissionais de 


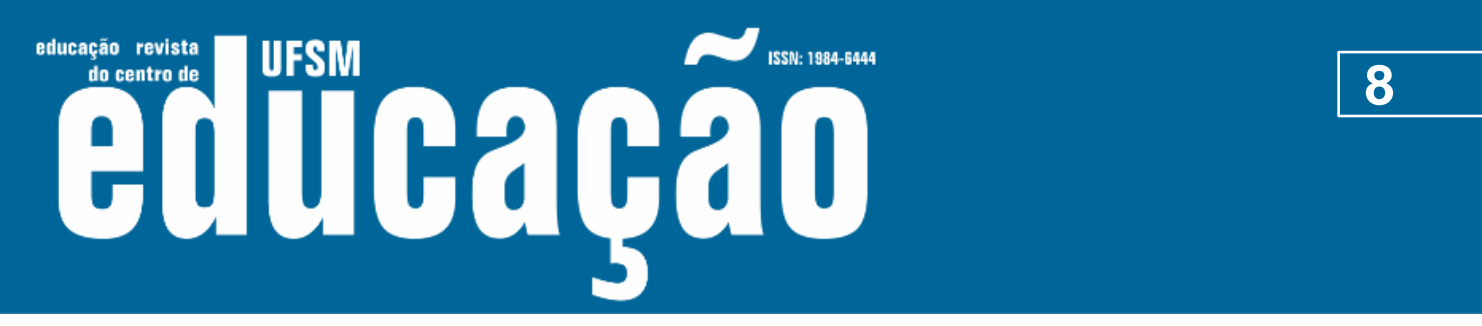

ISSN: 1984-6444 | http://dx.doi.org/10.5902/1984644443450

\section{Análise dos dados}

Os dados coletados foram organizados em planilhas do Software Microsoft Excel, de forma codificada conforme a natureza das variáveis. Posteriormente, as variáveis foram analisadas através da estatística descritiva por meio do software Statistical Package for Social Sciences (SPSS).

\section{Aspectos éticos}

Cumpriu-se todas as prerrogativas das diretrizes da Resolução 466/12 em relação à pesquisa com seres humanos (BRASIL, 2012). A coleta de dados teve início após aprovação do projeto de pesquisa pelo Comitê de Ética em Pesquisa da UFCSPA, sob parecer de número 1.876.126. Os participantes foram informados sobre os objetivos do estudo e orientados quanto ao sigilo das informações individuais. Assegurou-se ao participante o direito de interromper sua participação a qualquer momento da coleta, livre de prejuízos ou necessidade de justificativa.

\section{Resultados}

\section{Perfil sociodemográfico dos participantes}

Na tabela abaixo, apresenta-se o perfil sociodemográfico dos 632 discentes de graduação e de pós-graduação que responderam ao questionário.

Tabela 1 - Dados sociodemográficos

n $\%$

\begin{tabular}{lcc|} 
Sexo & \multicolumn{2}{l}{} \\
\hline Feminino & 416 & $65,8 \%$ \\
Masculino & 186 & $29,4 \%$ \\
Omissos & 30 & $4,7 \%$ \\
Raça & & \\
\hline Branca & 540 & $85,4 \%$ \\
\hline
\end{tabular}




\section{WFM

ISSN: 1984-6444 | http://dx.doi.org/10.5902/1984644443450

\begin{tabular}{|c|c|c|}
\hline Preta & 37 & $5,9 \%$ \\
\hline Parda & 43 & $6,8 \%$ \\
\hline Amarela & 2 & $0,3 \%$ \\
\hline Indígena & 2 & $0,3 \%$ \\
\hline Outras & 2 & $0,3 \%$ \\
\hline Omissos & 6 & 0,9 \\
\hline \multicolumn{3}{|l|}{ Estado Civil } \\
\hline Solteiro & 543 & $85,9 \%$ \\
\hline Casado & 56 & $8,9 \%$ \\
\hline Divorciado & 5 & $0,8 \%$ \\
\hline Viúvo & 1 & $0,001 \%$ \\
\hline Outros & 18 & $2,8 \%$ \\
\hline Omissos & 9 & $1,4 \%$ \\
\hline \multicolumn{3}{|l|}{ Crença } \\
\hline Budismo & 5 & $0,8 \%$ \\
\hline Candomblé & 4 & $0,6 \%$ \\
\hline Católica & 156 & $24,7 \%$ \\
\hline Espírita & 77 & $12,2 \%$ \\
\hline Evangélica & 49 & $7,8 \%$ \\
\hline Islamismo & 3 & $0,5 \%$ \\
\hline Judaísmo & 0 & $0 \%$ \\
\hline Protestante & 10 & $1,6 \%$ \\
\hline Umbanda & 8 & $1,3 \%$ \\
\hline Não se aplica & 304 & $48,1 \%$ \\
\hline Omissos & 16 & $2,5 \%$ \\
\hline \multicolumn{3}{|l|}{ Renda } \\
\hline 1 salário mínimo & 29 & $4,6 \%$ \\
\hline 2 a 3 salários mínimos & 153 & $24,2 \%$ \\
\hline
\end{tabular}




\section{. WFM

ISSN: 1984-6444 | http://dx.doi.org/10.5902/1984644443450

Até 5 salários mínimos

158

$25 \%$

Entre 5 e 10 salários mínimos

182

$28,8 \%$

Mais de 10 salários mínimos

76

$12 \%$

Mais de 20 salários mínimos

22

$3,5 \%$

Omissos

12

$1,9 \%$

\section{Série do Curso}

$1^{\text {a }}$ série

302

$47,8 \%$

$2^{\text {a }}$ série

184

$29,1 \%$

$3^{\text {a }}$ série

117

$18,5 \%$

$4^{a}$ série

18

$2,8 \%$

$5^{\text {a }}$ série

2

$0,3 \%$

$6^{\text {a }}$ série

4

$0,6 \%$

Omissos

5

$0,8 \%$

\section{Curso}

Enfermagem

Psicologia

Informática Biomédica

Física Médica

Nutrição

Biomedicina Diurno

Fisioterapia

Gestão em Saúde

Fonoaudiologia

Tecnologia de Alimentos

Biomedicina Noturno

Medicina

Farmácia

Pós Graduação Stricto Sensu 


\section{Hsm

ISSN: 1984-6444 | http://dx.doi.org/10.5902/1984644443450

em Enfermagem

Gastronomia 14

$2,2 \%$

Química Medicinal

\section{7}

$1,1 \%$

Toxicologia Analítica

5

$0,8 \%$

Programa de Pós-graduação Stricto Sensu em Ciências da

Reabilitação

Fonte: dados da pesquisa. (inserir ano)

\section{Compreensão dos discentes sobre a definição de espiritualidade}

A segunda etapa do questionário, refere-se às questões específicas sobre o tema espiritualidade. A primeira questão desta etapa é sobre o entendimento dos acadêmicos sobre espiritualidade, na qual se obteve os seguintes resultados (Tabela 2):

Tabela 2 - Definições de espiritualidade

\begin{tabular}{|c|c|c|}
\hline Definição & $\mathbf{N}$ & $\%$ \\
\hline $\begin{array}{l}\text { A transcendência entre o homem e a natureza, a arte e o } \\
\text { universo. }\end{array}$ & 193 & $30,5 \%$ \\
\hline $\begin{array}{l}\text { Uma força unificadora que não tem como propósito } \\
\text { aumentar a vida de uma pessoa, mas facilitar o seu } \\
\text { desenvolvimento. }\end{array}$ & 174 & $27,5 \%$ \\
\hline Um significado para a vida. & 122 & $19,3 \%$ \\
\hline É a transcendência entre o homem e Deus & 67 & $10,6 \%$ \\
\hline $\begin{array}{l}\text { Espiritualidade, religião e religiosidade são usadas como } \\
\text { sinônimos }\end{array}$ & 59 & $9,3 \%$ \\
\hline Omissos & 17 & $2,6 \%$ \\
\hline
\end{tabular}

Fonte: dados da pesquisa. (inserir ano) 


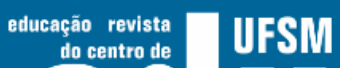

ISSN: 1984-6444 | http://dx.doi.org/10.5902/1984644443450

\section{Perspectiva dos alunos sobre o tema espiritualidade na formação profissional}

Na sequência, tem-se 9 questões do tipo Likert com pontuação de 1 a 5 . No quadro abaixo (Quadro 1), serão apresentados os resultados referentes a essas questões, incluindo as porcentagens proporcionais ao número total da amostra.

Quadro 1 - Dados dos questionários

\begin{tabular}{|c|c|c|c|c|c|}
\hline Questão & $\begin{array}{c}\mathbf{1} \\
\text { Discordo } \\
\text { totalmente }\end{array}$ & $\begin{array}{c}\mathbf{2} \\
\text { Discordo }\end{array}$ & $\begin{array}{c}\mathbf{3} \\
\text { Indiferente }\end{array}$ & $\begin{array}{c}4 \\
\text { Concordo }\end{array}$ & $\begin{array}{c}\mathbf{5} \\
\text { Concordo } \\
\text { totalmente }\end{array}$ \\
\hline $\begin{array}{l}\text { 1. É pertinente abordar } \\
\text { aspectos relacionados à } \\
\text { "espiritualidade" na formação } \\
\text { acadêmica. }\end{array}$ & $5,2 \%$ & $6,6 \%$ & $22 \%$ & $43,5 \%$ & $22,2 \%$ \\
\hline $\begin{array}{l}\text { 2. É pertinente abordar o tema } \\
\text { fé/espiritualidade na formação } \\
\text { acadêmica. }\end{array}$ & $5,2 \%$ & $12,3 \%$ & $24,7 \%$ & $40,5 \%$ & $16,6 \%$ \\
\hline \multirow{3}{*}{$\begin{array}{l}\text { 3. Os docentes de seu Curso } \\
\text { abordaram temas sobre } \\
\text { crenças religiosas ou } \\
\text { espirituais nas atividades } \\
\text { curriculares. Se sim, em qual } \\
\text { ano da graduação? }\end{array}$} & $34,5 \%$ & $35,6 \%$ & $18,5 \%$ & $18,2 \%$ & $2,2 \%$ \\
\hline & $1 \%$ ano & $2^{\circ}$ ano & 3ㅇ ano & $4^{\circ}$ ano & 5\%/6 ano \\
\hline & $41,9 \%$ & $22 \%$ & $9,2 \%$ & $1,1 \%$ & $0,8 \%$ \\
\hline $\begin{array}{l}\text { 4. A formação universitária } \\
\text { atual fornece informações } \\
\text { suficientes para que os } \\
\text { acadêmicos consigam abordar } \\
\text { as crenças religiosas ou } \\
\text { espirituais dos pacientes. }\end{array}$ & $23,3 \%$ & $47,8 \%$ & $18,4 \%$ & $9,5 \%$ & $0,5 \%$ \\
\hline $\begin{array}{l}\text { 5. Sinto-me preparado para } \\
\text { abordar aspectos relacionados } \\
\text { à "espiritualidade" com os } \\
\text { pacientes. }\end{array}$ & $14,4 \%$ & $34,5 \%$ & $19,1 \%$ & $25 \%$ & $4,7 \%$ \\
\hline $\begin{array}{l}\text { 6. Participei de atividade de } \\
\text { formação sobre a relação } \\
\text { "Saúde e Espiritualidade". }\end{array}$ & $78,6 \%$ & $10,1 \%$ & $7,6 \%$ & $2,5 \%$ & $0,5 \%$ \\
\hline Questão & $\begin{array}{c}1 \\
\text { Não } \\
\text { considero } \\
\text { importante } \\
\text { a } \\
\text { abordagem } \\
\text { do tema }\end{array}$ & $\begin{array}{c}\mathbf{2} \\
\text { Como um } \\
\text { tema } \\
\text { opcional } \\
\text { (somente } \\
\text { como } \\
\text { disciplina } \\
\text { eletiva) }\end{array}$ & $\begin{array}{c}\mathbf{3} \\
\text { Como um } \\
\text { tema } \\
\text { opcional } \\
\text { (somente } \\
\text { como } \\
\text { atividades } \\
\text { de } \\
\text { extensão/ }\end{array}$ & $\begin{array}{c}\mathbf{4} \\
\text { Como um } \\
\text { tema } \\
\text { opcional } \\
\text { (disciplina } \\
\text { eletiva/PD } \\
\mathrm{Cl} \text { e } \\
\text { atividades } \\
\text { de }\end{array}$ & $\begin{array}{c}\mathbf{5} \\
\text { Como um } \\
\text { tema } \\
\text { obrigatório } \\
\text { (disciplina } \\
\text { obrigatória) }\end{array}$ \\
\hline
\end{tabular}




\section{Fism oltibapá}

ISSN: 1984-6444 | http://dx.doi.org/10.5902/1984644443450

\begin{tabular}{|c|c|c|c|c|c|}
\hline & & & pesquisa) & $\begin{array}{l}\text { extensão/ } \\
\text { pesquisa }\end{array}$ & \\
\hline $\begin{array}{l}\text { 7. Considero importante } \\
\text { abordar questões sobre } \\
\text { espiritualidade na formação do } \\
\text { profissional da saúde: }\end{array}$ & $5,5 \%$ & $19,1 \%$ & $7,3 \%$ & $45,1 \%$ & $20,9 \%$ \\
\hline Questão & $\begin{array}{c}1 \\
\text { Eu não } \\
\text { busco } \\
\text { conhecime } \\
\text { ntos }\end{array}$ & $\begin{array}{l}\mathbf{2} \\
\text { Da minha } \\
\text { própria } \\
\text { religião }\end{array}$ & $\begin{array}{c}3 \\
\text { Docentes } \\
\text { de minha } \\
\text { Universidad } \\
\text { e }\end{array}$ & $\begin{array}{c}\mathbf{4} \\
\text { Leitura de } \\
\text { livros e } \\
\text { artigos } \\
\text { científicos/ } \\
\text { extensão/ } \\
\text { pesquisa }\end{array}$ & $\begin{array}{c}\mathbf{5} \\
\text { Palestras } \\
\text { que } \\
\text { abordam o } \\
\text { tema }\end{array}$ \\
\hline $\begin{array}{l}\text { 8. Busco conhecimentos sobre } \\
\text { o tema "Saúde e } \\
\text { Espiritualidade" por meio de: }\end{array}$ & $33,4 \%$ & $20,6 \%$ & $2,1 \%$ & $24,7 \%$ & $16,1 \%$ \\
\hline Questão & $\begin{array}{c}1 \\
\text { Desconfort } \\
\text { o com o } \\
\text { tema }\end{array}$ & $\begin{array}{c}\mathbf{2} \\
\text { Medo de } \\
\text { impor } \\
\text { pontos de } \\
\text { vista } \\
\text { religiosos } \\
\text { aos } \\
\text { pacientes, } \\
\text { medo de } \\
\text { ofender } \\
\text { os } \\
\text { pacientes }\end{array}$ & $\begin{array}{c}3 \\
\text { Medo de } \\
\text { que meus } \\
\text { colegas } \\
\text { não } \\
\text { aprovem }\end{array}$ & $\begin{array}{l}4 \\
\text { Não faz } \\
\text { parte do } \\
\text { meu } \\
\text { trabalho }\end{array}$ & $\begin{array}{l}\mathbf{5} \\
\text { Não faz } \\
\text { parte do } \\
\text { cuidado }\end{array}$ \\
\hline $\begin{array}{l}\text { 9. Os motivos pelos quais não } \\
\text { abordaria espiritualidade com } \\
\text { os pacientes: }\end{array}$ & $11,1 \%$ & $59,3 \%$ & $2,2 \%$ & $14,6 \%$ & $3,2 \%$ \\
\hline
\end{tabular}

Fonte: dados da pesquisa. (inserir ano)

\section{Discussão}

A maioria da amostra (87,9\%) entende a espiritualidade conforme a definição proposta pela literatura, ou seja, como uma conexão com o universo, um propósito de vida independente de religião e uma busca pessoal pelo sentido e significado da vida (KOENIG, 2001; SAAD, 2001). Em contraponto, 9,3\% entende espiritualidade, religião e religiosidade como sinônimos, embora a literatura aponte o contrário (SOUZA, MAFTUM E BAIS, 2008).

Sabe-se que o ser humano deve ser compreendido em sua integralidade, conforme os princípios do Sistema Único de Saúde, e a dimensão espiritual é parte 


\section{-

ISSN: 1984-6444 | http://dx.doi.org/10.5902/1984644443450

constatou que em nenhum deles constava conteúdos sobre espiritualidade (JORDAN; BARBOSA, 2019). Evidencia-se que há uma fragilidade em contemplar a integralidade do cuidado dos pacientes tanto na graduação quanto na pós-graduação.

É necessário o desenvolvimento de mais estudos para avaliar quais os melhores recursos, estratégias e o momento ideal para abordar o tema da espiritualidade nos cursos de graduação e de pós-graduação (DAMIANO et al, 2018). Ressalta-se a relevância da implementação de atividades práticas, visto que a literatura afirma que resulta em uma significativa diferença no desempenho da anamnese espiritual realizada pelos estudantes (MUSICK et al, 2003).

\section{Conclusões}

Conclui-se que os discentes dos diferentes cursos e séries reconhecem a relevância do estudo da espiritualidade no âmbito da saúde, entretanto, a carência deste conteúdo durante a formação acadêmica resulta em desconhecimento e insegurança para abordar o tema com os pacientes. À vista disso, necessita-se de inclusão de disciplinas direcionadas a esse assunto, a fim de promover um cuidado integral e humanístico.

Ao considerarmos a importância dos aspectos espirituais no processo saúdedoença, tem-se que o profissional de saúde deve buscar compreender e respeitar os significados individuais e as diferentes formas de expressar a espiritualidade. Ressalta-se que a espiritualidade é uma tecnologia leve e sem custo para a assistência, que apresenta evidentes contribuições para o enfrentamento de doenças e para o bem-estar dos indivíduos e, portanto, precisa ser difundida e abordada durante a formação acadêmica por meio de atividades eletivas e obrigatórias. A abordagem transversal da espiritualidade nas disciplinas inespecíficas ao tema é a realidade, e não garante $o$ aprofundamento necessário que 0 aluno precisa para se sentir apto na aplicação do conhecimento na prática.

Evidencia-se a emergente necessidade da realização de novas pesquisas sobre a percepção dos discentes em relação ao tema espiritualidade e de estudos que avaliem quais as metodologias de ensino-aprendizagem apropriadas para abordagem 


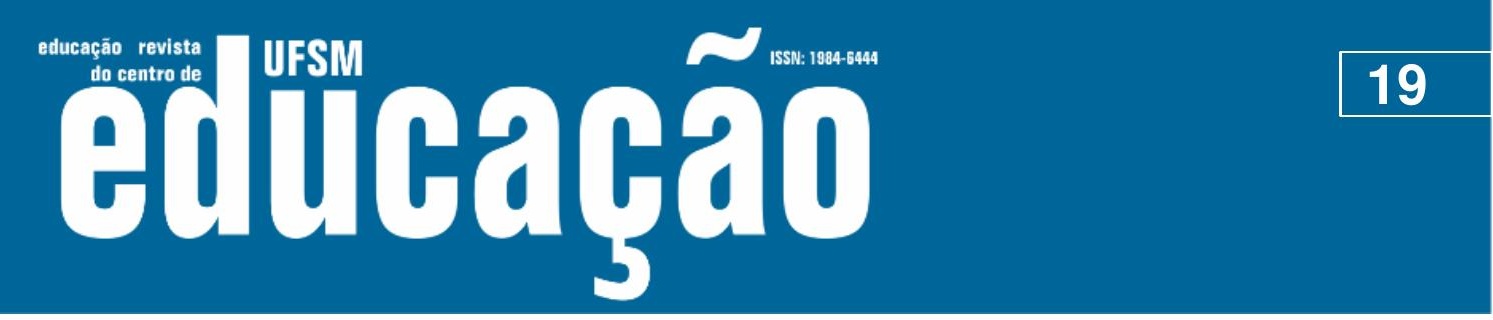

ISSN: 1984-6444 | http://dx.doi.org/10.5902/1984644443450

do assunto, além de mensurar o impacto da espiritualidade na assistência em saúde e na formação do profissional.

Por fim, tem-se como limitação o fato deste estudo ter sido realizado apenas sob a perspectiva dos alunos, logo, sugere-se que outras pesquisas sejam desenvolvidas para conhecer a percepção dos docentes acerca da pertinência da inserção do tema nos currículos de graduação e de pós-graduação. Há também a necessidade de avaliar o conhecimento dos docentes sobre o assunto, em função de que os próprios professores podem não ter tido formação prévia sobre a espiritualidade na saúde, influenciando diretamente na possibilidade desse tema ser abordado transversalmente e em disciplinas específicas. Além disso, entende-se que seria relevante estudar a população longitudinalmente, a fim de compreender como as opiniões dos alunos sobre o assunto poderiam se modificar ao longo da formação.

\section{Referências}

AGUIAR, Paulo Rogerio; CAZELLA, Silvio César; COSTA, Marcia Rosa. A Religiosidade/Espiritualidade dos Médicos de Família: Avaliação de Alunos da Universidade Aberta do SUS (UNA-SUS). Rev. bras. educ. med., Rio de Janeiro, v. 41, n. 2, p. 310-319, $2017 . \quad$ Disponível em: http://www.scielo.br/scielo.php?script=sci_arttext\&pid=S0100$55022017000200310 \&$ Ing=en\&nrm=iso. Acesso em 25 Mar. 2020.

ALVES, Joseane de Souza; JUNGES, José Roque; LÓPEZ, Laura Cecília. A dimensão religiosa dos usuários na prática do atendimento à saúde: percepção dos profissionais da saúde. Mundo saúde, v.34, n.2, p. 430-436, 2010. Disponível em: http://www.saocamilo-sp.br/pdf/mundo_saude/79/430a436.pdf. Acesso em: 05 Abr. 2020.

ASSOCIATION OF AMERICAN MEDICAL COLLEGES. Report I: Learning objectives for medical student education: guidelines for medical schools. American Association of Medical Colleges, v.74, n.1, p.13-18, 1998. Disponível em: https://www.ncbi.nlm.nih.gov/pubmed/9934288. Acesso em: 01 abr. 2020.

BRASIL. Resolução n 466, de 12 de dezembro de 2012. Diário Oficial da República Federativa do Brasil, Brasília, DF, 2013. 


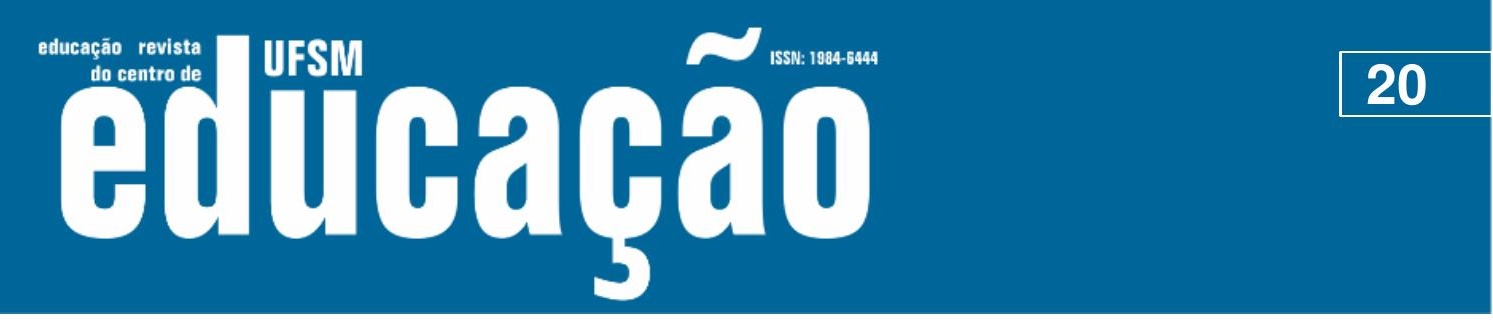

ISSN: 1984-6444 | http://dx.doi.org/10.5902/1984644443450

CASALETTI, Camilla et al. Espiritualidade, saúde e suas aplicações práticas: desenvolvimento do Programa de Saúde, Espiritualidade e Religiosidade. HU Revista, v. 44, n. 4, p. 507-514, 2020. Disponível em: https://periodicos.ufjf.br/index.php/hurevista/article/view/26389. Acesso em: 05 Abr. 2020.

COPELLO, Luciane Escobar; PEREIRA, Adriana Dall'Asta; FERREIRA,Carla Lizandra de Lima. Espiritualidade e religiosidade: importância para o cuidado de enfermagem de paciente em processo de adoecimento. Disciplinarum Scientia. Série: Ciências da Saúde, Santa Maria, n. 2, p. 183-199, 2018. Disponível em: https://periodicos.ufn.edu.br/index.php/disciplinarumS/article/view/2504. Acesso em: 05 Abr. 2020.

COSTA, Milena Silva et al. Espiritualidade e religiosidade: saberes de estudantes de medicina. Rev. Bioét., Brasília, n. 2, p. 350-358, 2019. Disponível em: http://www.scielo.br/scielo.php?script=sci_arttext\&pid=S1983-

80422019000200350\&lng=en\&nrm=iso. Acesso em: 06 Abr. 2020.

DAL-FARRA, Rossano André; GEREMIA, César. Educação em saúde e espiritualidade: proposições metodológicas. Rev. bras. educ. med., Rio de Janeiro, v. 34, n. 4, p. 587-597, 2010. Disponível em:http://www.scielo.br/scielo.php?script=sci_arttext\&pid=S0100-

55022010000400015\&lng=en\&nrm=iso. Acesso em: 06 Abr. 2020.

DAMIANO, R. F.; LUCCHETTI, A. L. G.; LUCCHETTI, G. Ensino de "saúde e espiritualidade" na graduação em medicina e outros cursos da área de saúde. HU Revista, v. 44, n. 4, p. 515-525, 2020. Disponível em: https://periodicos.ufjf.br/index.php/hurevista/article/view/25928/20212. Acesso em: 06 Abr. 2020.

ESPINHA, Daniele Corcioli Mendes et al. Opinião dos estudantes de enfermagem sobre saúde, espiritualidade e religiosidade. Rev. Gaúcha Enferm., Porto Alegre, n. 4, p. 98-106, 2013. Disponível em: http://www.scielo.br/scielo.php?script=sci_arttext\&pid=S1983-

14472013000400013\&lng=en\&nrm=iso. Acesso em: 06 Abr. 2020.

FERREIRA, Alberto Gorayeb de Carvalho; OLIVEIRA Janaíne Aline Camargo de; JORDÁN, Arturo de Pádua Walfrido. Educação em saúde e espiritualidade: uma proposta de transversalidade na perspectiva do estudante. Interdisciplinary Journal of Health Education, v.1, n1, 2016. Disponível em: https://ijhe.emnuvens.com.br/ijhe/article/view/1/3. Acesso em: 04 Abr. 2020. 


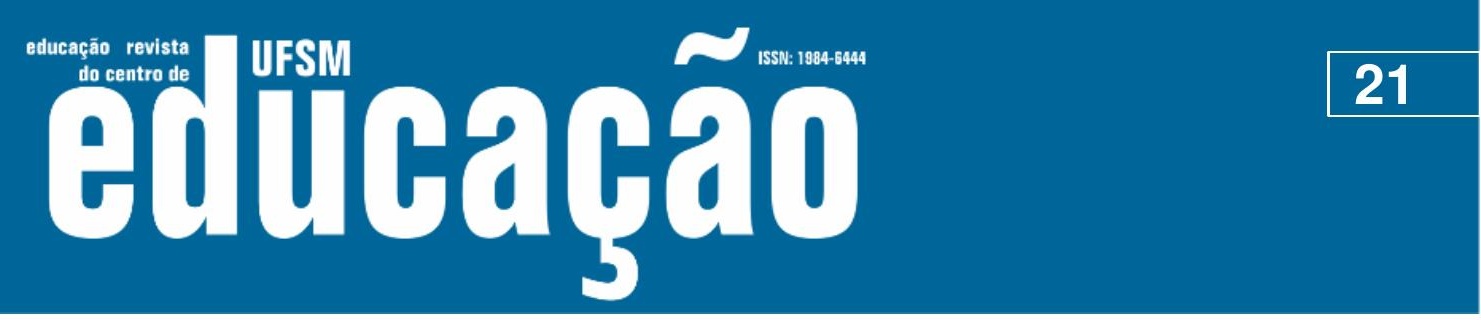

ISSN: 1984-6444 | http://dx.doi.org/10.5902/1984644443450

FERREIRA, Tassiani Turra et al. Percepção de Acadêmicos de Medicina e de Outras Áreas da Saúde e Humanas (Ligadas à Saúde) sobre as Relações entre Espiritualidade, Religiosidade e Saúde. Rev. bras. educ. med., Brasília, v. 42, n. 1, p. 67-74, 2018. $\quad$ Disponível em http://www.scielo.br/scielo.php?script=sci_arttext\&pid=S0100-

55022018000100067\&lng=pt\&nrm=iso. Acesso em: 06 Abr. 2020.

GUSSI, Maria Aparecida; DYTZ, Jane Lynn Garrison. Religião e espiritualidade no ensino e assistência de enfermagem. Rev. bras. enferm., Brasília, v. 61, n. 3, p. 337384, 2008.2 Disponível em: http://www.scielo.br/scielo.php?script=sci_arttext\&pid=S0034-

71672008000300017\&lng=en\&nrm=iso. Acesso em: 06 Abr. 2020.

HARMUCH, Camila; CAVALCANTE, Marília Daniella Machado Araújo; ZANOTIJERONYMO, Daniela Viganó. Religião E Espiritualidade No Ensino e Assistência de Enfermagem na visão dos estudantes: uma revisão. Rev. UNINGÁ, Maringá, n. 2, p. 243-254, 2019. Disponível em: http://34.233.57.254/index.php/uninga/article/view/938/1917. Acesso em: 6 Abr. 2020

HENZ, Kaena Garcia; NASCIMENTO, Filipe Rodrigues do; SILVA, Lucas Henrique Rodrigues da, et al. Liga Acadêmica De Saúde E Espiritualidade - Liase in Ligas acadêmicas: definições, experiências e conclusões. Porto Alegre: UFRGS, p. 5664, 2018. Disponível em: https://www.lume.ufrgs.br/bitstream/handle/10183/175031/001064187.pdf?sequence $=1$. Acesso em: 04 Abr.2020.

JORDAN, Arturo de Pádua Walfrido; BARBOSA, Leopoldo Nelson Fernandes. Espiritualidade e Formação nos Programas de Residência em Saúde de uma Cidade no Nordeste Brasileiro. Rev. bras. educ. med., Brasília, v. 43, n. 3, p. 82-90, 2019. Disponível em: http://www.scielo.br/scielo.php?script=sci_arttext\&pid=S010055022019000300082\&lng=en\&nrm=iso. Acesso em: 05 Abr. $20 \overline{2} 0$

KOENIG, Harold G.; LARSON, David B.; LARSON, Susan S. Religion and coping with serious medical illness. The Annals of Pharmacotherapy, v, 35, 352-58, 2001. Disponível em: https://www.ncbi.nlm.nih.gov/pubmed/11261534. Acesso em 25 Mar. 2020.

KOENIG, Harold G. Espiritualidade no Cuidado com o Paciente. Fé Editora, 2005.

KOENIG, Harold G. Religion, spirituality, and health: the research and clinical implications. ISRN Psychiatry, 1-33, 2012. Disponível em: https://www.ncbi.nlm.nih.gov/pubmed/23762764. Acesso em 20 Mar.2020.

LUCCHETTI, Giancarlo et al; Spirituality in medical education: new initiatives in Brazil. Clin Teach, n 3, 213-213, 2011. Disponível em: https://bit.ly/2UfhLjQ. Acesso em: 05 Abr.2020 


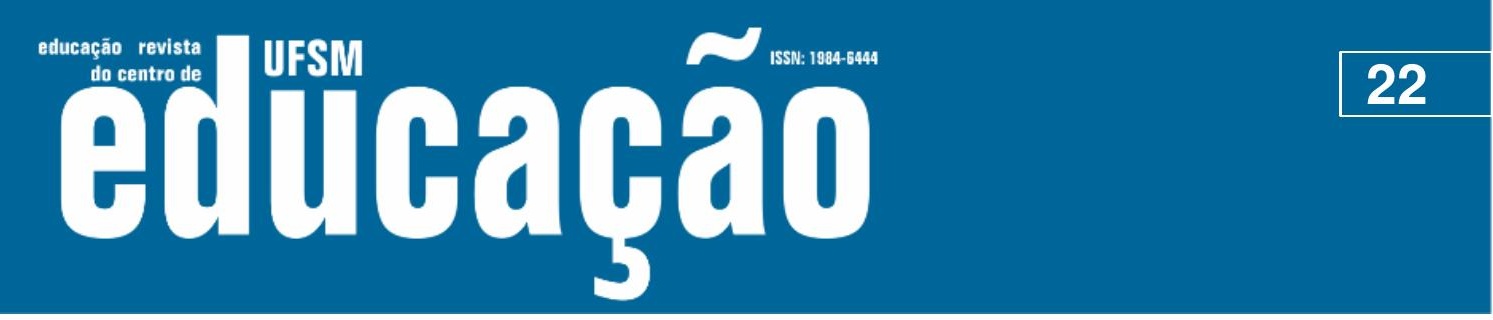

ISSN: 1984-6444 | http://dx.doi.org/10.5902/1984644443450

LUCCHETTI, Giancarlo et al.Medical students, spirituality and religiosity-results from the multicenter study SBRAME. BMC Med Educ, v.13, 2013. Disponível em: https://www.ncbi.nlm.nih.gov/pubmed/24314327. Acesso em: 26 Mar. 2020

LUCCHETTI, Giancarlo et al. Spirituality and health in the curricula of medical schools in Brazil. BMC Med Educ, v.12, 2012. Disponível em: https://bmcmededuc.biomedcentral.com/articles/10.1186/1472-6920-12-78. Acesso em: 25 Mar .2020

MOREIRA-ALMEIDA, Alexander; LOTUFO NETO, Francisco; KOENIG, Harold G. Religiousness and mental health: a review. Rev. Bras. Psiquiatr., São Paulo, v. 28, n. 3, p. 242-250, 2006. Disponível em: http://www.scielo.br/scielo.php?script=sci_arttext\&pid=S1516-

44462006000300018\&lng=en\&nrm=iso. Acesso em: 06 Abr. 2020.

MUSICK, David W; CHEEVER, Todd R; QUINLIVAN, Sue; NORA, Lois Margaret. Spirituality in medicine: a comparison of medical students' attitudes and clinical performance. Acad Psychiatry, v.27, n.2, p. 67-73, 2003. Disponível em: https://www.ncbi.nlm.nih.gov/pubmed/12824105. Acesso em: 06 Abr. 2020

NASCIMENTO, Lucila Castanheira et al. Espiritualidade e religiosidade na perspectiva de enfermeiros. Texto contexto - enferm., Florianópolis, v. 22, n. 1, p. 52-60, 2013. Disponível em: http://www.scielo.br/scielo.php?script=sci_arttext\&pid=S010407072013000100007\&lng=en\&nrm=iso. Acesso em: 06 Abr. 2020.

OLIVEIRA, Fabiano Fernandes de; FRAZILI, Rosana Tupinambá Viana. Espiritualidade: seu significado no contexto do acadêmico de enfermagem. REENVAP, Lorena, n. 02, 2012. Disponível em:https://docplayer.com.br/19122419Espiritualidade-seu-significado-no-contexto-do-academico-de-enfermagem.html.

Acesso em: 03 Abr. 2020.

OLIVEIRA, Raquel Aparecida de. Saúde e espiritualidade na formação profissional em saúde, um diálogo necessário.Rev Fac Ciênc Méd Sorocaba, v. 19, n. 2, p. 54-55, 2017. Disponível em: https://revistas.pucsp.br/RFCMS/article/view/32819. Acesso em: 06 Abr. 2020.

ORGANIZAÇÃO MUNDIAL DA SAÚDE. National cancer control programmes: policies and managerial guidelines. Geneve: OMS, 2002.

PUCHALSKI, Christina M et al. Spirituality and health: the development of a field. Acad Med, v.89, n.1, p.1-7, 2014. Disponível em: https://www.ncbi.nlm.nih.gov/pubmed/24280839. Acesso em: 20 Mar. 2020. 


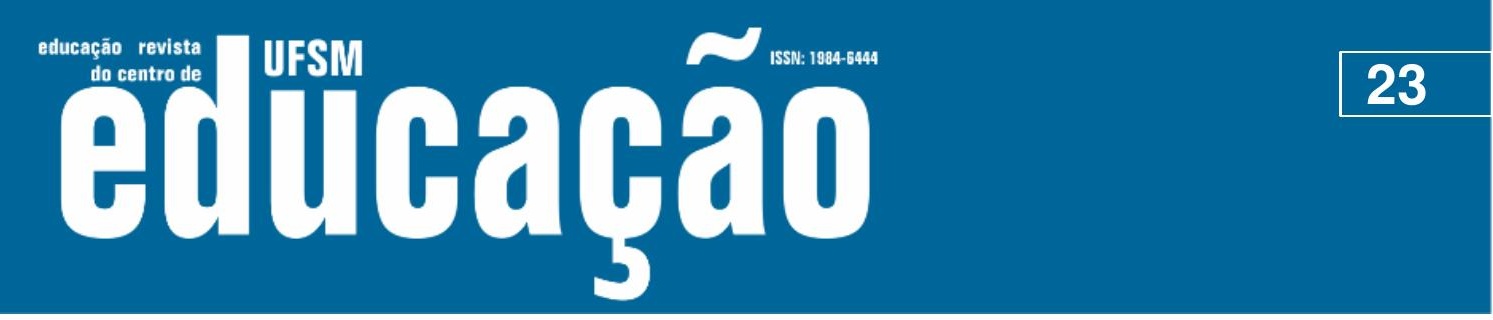

ISSN: 1984-6444 | http://dx.doi.org/10.5902/1984644443450

RADDATZ, Juliana Schmidt; MOTTA, Roberta Fin; ALMINHANA, Letícia Oliveira. Religiosidade/Espiritualidade na Prática Clínica: Círculo Vicioso entre Demanda e Ausência de Treinamento. Psico-USF, Campinas, n. 4, p. 699-709, 2019. Disponível em: $\quad$ http://www.scielo.br/scielo.php?script=sci_arttext\&pid=S1413$82712019000400699 \&$ Ing=en\&nrm=iso. Acesso em 06 Abr. 2020.

REGINATO, Valdir; BENEDETTO, Maria Auxiliadora Craice De; GALLIAN, Dante Marcello Claramonte. Espiritualidade e Saúde: Uma experiência na graduação em Medicina e Enfermagem. Trab. educ. saúde, Rio de Janeiro, n. 1, p. 237-255, 2016. Disponível em: http://www.scielo.br/scielo.php?script=sci_arttext\&pid=S198177462016000100237\&lng=en\&nrm=iso. Acesso em: 06 Abr. 2020.

RIGHETTI, Sabine. Pode a fé curar?. SBPC, 2005. Disponível em: https://bit.ly/2OWbP9L. Acesso em: 01 Abr. 2020

SÁ, Ana Cristina. Reflexão sobre o cuidar em Enfermagem: uma visão do ponto de vista da espiritualidade humana e da atitude crística. O Mundo da Saúde São Paulo, n 2, 205-217, 2009. Disponível em: http://www.saocamilosp.br/pdf/mundo_saude/67/205a217.pdf. Acesso em 06 Abr. 2020.

SAAD, Marcelo; MASIERO, Danilo; BATTISTELLA, Linamara Rizzo. Espiritualidade baseada em evidências. Acta Fisiátrica, v.8, n.3, 107-122, 2001. Disponível em: http://www.actafisiatrica.org.br/detalhe_artigo.asp?id=314. Acesso em: 20 Mar. 2020.

SILVA, Maria Beatriz Andrade Marques; AMARAL, Lorena Borges Cambraia Barreiros; ALMEIDA, Rinaldo Borges de; GROSSMANN Soraya de Matos Camargo. Espiritualidade e Saúde: Estudo Caso-Controle. Revista da Universidade Vale do Rio Verde, Três Corações, v. 14, n. 2, p. 1201-1209, 2016. Disponível em: http://periodicos.unincor.br/index.php/revistaunincor/article/view/3456. Acesso em: 06 Abr. 2020.

SIMÕES, Naiane Dias et al. Espiritualidade e saúde: experiência de uma disciplina na graduação de enfermagem. Revista de Enfermagem da UFSM, [S.I.], v. 8, n. 1, p. 181-191, 2018. Disponível em: https://periodicos.ufsm.br/reufsm/article/view/25038. Acesso em: 05 Abr. 2020.

SOUZA, Janei Rabello; MAFTUM, Mariluci Alves; BAIS, Dulce Dirclair Huf. O cuidado de enfermagem em face do reconhecimento da crença e/ ou religião do paciente: percepções deestudantes de graduação. Online Brazilian Journal of Nursing, v. 7, n. 2, 2008. Disponível em: http://www.objnursing.uff.br/index.php/nursing/article/view/1525/375. Acesso em: 06 Abr. 2020.

SULMASY, Daniel P. Spirituality, Religion, and Clinical Care. Chest, v.135, n.6, p. 1634-1642, 2009. Disponível em: 


\section{W HEM \\ ISSN: 1984-6444

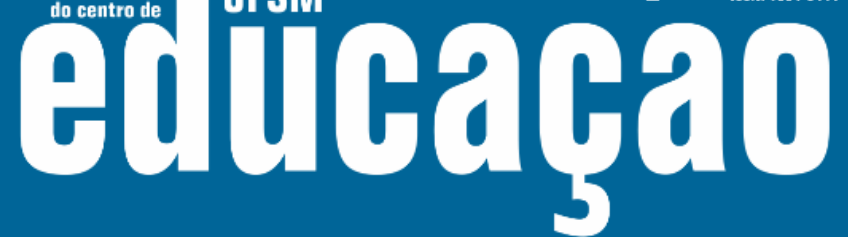

ISSN: 1984-6444 | http://dx.doi.org/10.5902/1984644443450

https://www.sciencedirect.com/science/article/abs/pii/S0012369209603706. Acesso em: 03 Abr.2020.

\section{(9) $(1) \Theta$}

This work is licensed under a Creative Commons Attribution-NonCommercial 4.0 International (CC BY-NC 4.0) 